\title{
Shipboard Helicopters, Sea Monsters, Safety ... and Engineering
}

\author{
Rob Langlois \\ Department of Mechanical and Aerospace Engineering \\ Carleton University
}

The development of maritime helicopters was initially motivated by the need to counter the new threat to ships posed by submarines. However, since their introduction, the role of maritime helicopters has steadily grown such that they are now an essential and integral part of maritime operations. Shipboard aircraft provide a flexible and fast means for accessing large off-shore areas. However, their effectiveness is dependent upon the ability to reliably operate them from ships and at-sea platforms in 'all' conditions. In particular, the Canadian theatre of operations is characterized by some of the most adverse sea conditions in the world. As a result, Canada has led innovation in shipboard helicopter operation and is recognized as a world leader in terms of capable maritime aviators, procedures, and equipment. Due to the versatility of maritime helicopters and the need for efficiency, there is a global trend towards operating larger helicopters from the decks of smaller ships in increasingly severe sea conditions. This further compounds the engineering challenges associated with ensuring consistent, safe, and reliable flight operations.

This presentation will overview key aspects of maritime helicopter use, highlight Canadian contributions to the field, and identify applied dynamics problems associated with the various phases of shipboard helicopter operation; including ondeck securing and traversing, rotor engage and disengage, launch and recovery, and shipboard postural stability. Since shipboard systems - both mechanical and human - experience loads generated by geometrically-nonlinear and timedependent ship motion, nonlinear and intermittent deck contact and sliding, time- and displacement-dependent aerodynamic forces, and a variety of securing forces resulting from nonlinear passive and active securing devices, the associated dynamics problems are rich and captivating. In fact, the nature of the problem usually renders conventional static, quasi-static, and frequency-domain analysis approaches inadequate for understanding the true nature of the dynamic interface. The various classes of problems and solution approaches will be discussed. The presentation will conclude with an overview of on-going research in the Carleton University Applied Dynamics Laboratory, which is contributing to a better understanding of the human/aircraft/ship dynamic interface. 